\title{
Sensing Properties of a Fabry-Perot Dielectric Structure and Dimer Nanoparticles
}

\author{
A. Polemi and K. L. Shuford \\ Department of Chemistry, Drexel University, 3141 Chestnut Street, Philadelphia, PA 19104, USA \\ Correspondence should be addressed to A. Polemi, alessia.polemi@drexel.edu
}

Received 30 November 2011; Accepted 18 January 2012

Academic Editor: Mustafa Çulha

Copyright (๑) 2012 A. Polemi and K. L. Shuford. This is an open access article distributed under the Creative Commons Attribution License, which permits unrestricted use, distribution, and reproduction in any medium, provided the original work is properly cited.

\begin{abstract}
We investigate the use of a Fabry-Perot dielectric structure combined with differently shaped nanoparticles for Surface Enhanced Raman Scattering. In particular, we show how an ideal two-layer Fabry-Perot configuration enhances the local surface field of silver nanoparticles positioned on the surface of the structure. We develop the concept using disc dimers and then extend the discussion to bowtie nanoparticles. The structure is excited by a single emitter, which couples to the nanoparticles through the dielectric layers, producing a wide aperture field that can be used to excite multiple dimers. We show how an array of nanoparticles can be properly arranged in order to increase the total scattering signal generated from the structure. The layered geometry produces robust field properties in between nanoparticles, making the overall sensing characteristics less sensitive to the interparticle seperation distance and incident polarization.
\end{abstract}

\section{Introduction}

In the recent years, the utilization of Surface Enhanced Raman Spectroscopy (SERS) has grown dramatically, demonstrating its power and utility as an analytical tool for the selective detection of molecules interacting with metal surfaces $[1,2]$. The SERS enhancement is often interpreted as the product of two contributions [3]: an electromagnetic (EM) enhancement mechanism and a chemical enhancement mechanism. It has been shown that the electromagnetic enhancement is dominant, and it produces the larger contribution [4-6]. This contribution is due to the localized surface plasmon resonance (LSPR), which excites the molecule under study when near a plasmonic surface. An LSPR occurs when the collective oscillation of conduction electrons in a metal nanoparticle is in resonance with the frequency of incident light. When the excitation field is resonant with a plasmon, the metal particle will emit coherent electromagnetic radiation. This radiation increases the local field intensity, $|E|$, that molecules feel when located near the plasmonic structure. It has been established that the Raman scattering enhancement scales approximately as
$|E|^{4}[6]$. Thus, the use of a proper plasmonic nanostructure is essential to maximize the electromagnetic effect, reaching typical values of SERS gain in the range $10^{5}-10^{6}$. On the other side, the chemical enhancement, which is primarily due to the excitation of adsorbate localized electronic resonances or metal-to-adsorbate charge-transfer resonances, does not usually exceed $10^{2}-10^{3}[7,8]$.

Both theoretical calculations and experiments have shown how the plasmon frequency and resulting EM field are extremely dependent on the nanoparticle composition, size, shape, and dielectric environment $[9,10]$. In particular, in a recent paper [11], we have investigated the use of a Fabry Perot (FP) dielectric structure to improve the local field coupled to a nanodisc dimer. The FP concept is well known in the literature and widely used in the radio frequency domain. In [11], we have used an FP planar dielectric structure formed by a superstrate with a high permittivity placed on a substrate with very low permittivity. This substrate/superstrate structure, once properly designed, has been demonstrated to establish a resonance condition, which maximizes antenna gain, radiation resistance, and radiation efficiency [12-14]. In particular, we have focused 
on dimer nanoparticles formed by a pair of silver discs placed on top of the double-layer FP structure. A quantum emitter, treated as a classical dipole [15], excites the dimer through the dielectrics by means of a wide aperture field generated on top of the superstrate. Since the source is linearly polarized, the aperture field is also linearly polarized [16], and it is used as a secondary feeding source for the dimer nanoparticles. In [11], we focused primarily on the far field properties of the system, described as an antenna at optical frequencies, and only briefly showed how this structure can be also used for sensing. In the present paper, we focus our attention on the sensing properties, with particular reference to SERS. Here, we will expand our investigation on the disc dimer and will explore the possibility of employing differently shaped nanoparticles, such as bowtie arrangement. At the same time, we will also investigate the effect of losses of realistic dielectric materials on the sensing performance. Then, we will discuss how disc dimers and bowtie nanoparticles can be arranged in an array configuration, within the wide aperture field provided by the FP structure, and how this improves the SERS gain. Finally, we will show how the FP structure is quite robust to changes of the source polarization, and how different orientations of the dipolar emitter can be used to increase the overall field enhancement.

\section{Fabry-Perot Cavity and Nanoparticles}

The Fabry-Perot dielectric structure is composed of two dielectric slabs (substrate/superstrate) with dielectric permittivity $\varepsilon_{r 1}$ and $\varepsilon_{r 2}$, respectively (see Figure 1 ). In order to obtain a substantial FP cavity effect, $\varepsilon_{r 2} \gg \varepsilon_{r 1}$, typically $\varepsilon_{r 2} \sim$ $\varepsilon_{r 1}$. Initially for simplicity, we assume that the substrate is free space $\left(\varepsilon_{r 1}=1\right)$, and the superstrate is a lossless material with permittivity $\varepsilon_{r 2}=10$. This assumption can be removed, and other materials can be used with permittivities that fulfill the FP requirements. We will show in the following sections how the use of realistic materials does not alter the general behavior of the structure. The structure is designed at the operating wavelength $\lambda=633 \mathrm{~nm}$. At this frequency, the substrate and the superstrate must have thickness $h_{1}=\lambda_{1} / 2=316.5 \mathrm{~nm}$ and $h_{2}=\lambda_{2} / 4=50 \mathrm{~nm}$, where $\lambda_{1}=\lambda / \sqrt{\varepsilon_{r 1}}$ and $\lambda_{2}=\lambda / \sqrt{\varepsilon_{r 2}}$ are the wavelengths in the dielectrics with permittivity $\varepsilon_{r 1}$ and $\varepsilon_{r 2}$, respectively, and $\lambda$ is the wavelength in free space. The substrate is grounded by a silver plate (optical constants taken from [17]), with a thickness greater than the skin depth in order to ensure total reflectivity. We have utilized silver for the sake of fabrication continuity (the nanoparticles are made of silver). The quantum emitter, represented here as a dipole oriented along $y$ with unit current and length of $5 \mathrm{~nm}$ (dipole moment $\bar{p}=5 \cdot 10^{-9} \hat{y} \mathrm{Cm}$ ), is placed in the substrate in a location that maximizes the electric field inside the cavity. For the ideal case of a perfectly conducting ground plane, this occurs when $h=h_{1} / 2=158.25 \mathrm{~nm}$. In our case, due to the presence of the silver ground plane, the ideal dipole position has been found to be $h=143 \mathrm{~nm}$ [11]. Note that in a real experiment, an external source excites the quantum emitter, which couples to the dimer through the FP structure. This could be implemented, for example, by

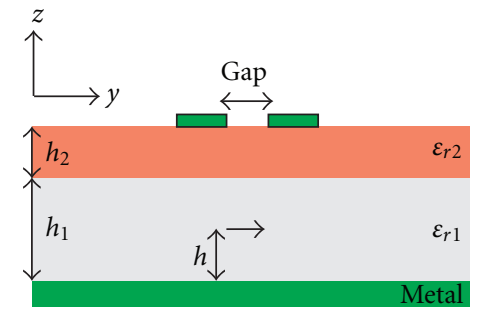

FIGURE 1: Reference geometry of the structure. The dipolar source is embedded in the substrate, which has permittivity $\varepsilon_{r 1}$ and height $h_{1}$. The superstrate is characterized by permittivity $\varepsilon_{r 2}$ and height $h_{2}$. The nanoparticles are placed on top of the superstrate.
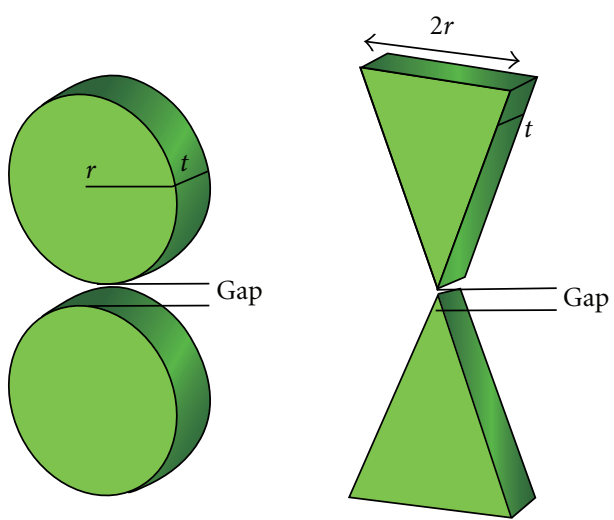

Figure 2: Geometry of the two nanoparticle configurations: disc dimer and bowtie structure.

means of a nanoemitter on top of a focusing plasmonic tip source. How this affects the FP excitation efficiency is not presented here but is being investigated currently.

2.1. Disc Dimers and Bowtie Nanoparticles. In [11], we have shown how the wide linearly polarized aperture field can be used as a secondary field source to feed a pair of disc nanoparticles, composed of silver with radius $r$, thickness $t$, and separation gap, placed on the top of the superstrate. Here, we want to investigate their sensing capability compared to a silver bowtie structure. The base of the triangle forming the bowtie is set to $2 r$, and the remaining dimensions are assigned in order to have the same total surface area as the disc dimer. The two nanoparticle configurations are shown in Figure 2.

Under the assumption that the Raman signal is only slightly shifted in frequency with respect to the source frequency, the SERS enhancement factor can be calculated as

$$
E F_{\text {SERS }}=\left|\frac{E}{E_{0}}\right|^{4},
$$

where the electric field around the particles $E$ and the source field $E_{0}$ are calculated at $\lambda=633 \mathrm{~nm}$ in this case. It is well known that the local field of the nanoparticle spikes in the proximity of its edges. When employing grid-based numerical simulations, the value of the field close to the 
metal boundary is sensitive to the size of the grid. For these reasons, we have introduced a more representative figure of merit, which is the average of the electric field over the particle surface area $[11,18,19]$. In particular, we define $E_{\text {surf }}$ as the total absolute field integrated over the surface $\left(S=S_{\text {disc }}, S_{\text {bowtie }}\right)$ of the nanoparticles accessible to molecules

$$
E_{\text {surf }}=\frac{1}{S} \int_{S}|\bar{E}(x, y, z)| d S,
$$

and we use this in place of $|E|$ in (1). The value of $E_{\text {surf will }}$ be notably less than the maximum surface field but will likely be more in line with what one might actually measure in an experiment. In order to obtain a consistent definition of $E F_{\text {SERS }}$, we equivalently define the source field $E_{0}$ in (1) as the average of the electric field over the same particle surface area as in (2), but produced by the dipole in free space, that is,

$$
E_{\text {surf }}^{0}=\frac{1}{S} \int_{S}\left|\overline{E_{0}}(x, y, z)\right| d S,
$$

where $\overline{E_{0}}(x, y, z)$ is the dipole source in the absence of the dielectric structure and of the nanoparticles. This allows us to compare the fields around the nanoparticles without including the effect of the spherical spreading factor associated with a dipole field. Thus, the SERS enhancement factor can be redefined as

$$
E F_{\text {SERS }}^{\text {avg }}=\left|\frac{E_{\text {surf }}}{E_{\text {surf }}^{0}}\right|^{4} .
$$

Notice that $E_{\text {surf }}^{0}$ differs for the disc dimer and the bowtie since the area where the surface integral is performed is geometrically different.

Calculations have been performed with CST Microwave Studio [20] using the time domain solver. Within this approach, Maxwell's equations are solved by performing a Finite Integration Technique [21], which relies on the discretization of the geometrical domain in terms of hexahedrons. In our case, the mesh size is set to $1 \mathrm{~nm}$. Perfect Matching Layer boundary conditions are applied to the walls of the domain box.

In Figure 3, we show the $E_{\text {surf }} / E_{\text {surf }}^{0}$ ratio for the disc dimer (blue line) and the bowtie (red line) on top of the FP structure, with the dimer axis aligned along the dipole source (see Figure 1). The ratio is presented as a function of the gap distance for a radius $r=40 \mathrm{~nm}$ (Figure 3(a)), and as a function of the radius $r$ when gap $=5 \mathrm{~nm}$ (Figure 3(b)). In general, the sensing properties of the disc dimer and the bowtie are very similar, except that the bowtie works slightly better when the value of $r$ increases. At this excitation wavelength, smaller particles of both varieties tend to generate larger fields as shown in Figure 3(b). Notice that the variation of $r$ affects the bowtie geometry as well, since both the particles are designed in order to have the same total surface area. Although one would expect that the bowtie geometry provides a larger field because of the sharp tips, we must stress again that we are presenting the integral of the electric field on the particle surface, not in the gap area. Presumably for many SERS applications, the molecules being sensed bind only to the metallic surface, negating most of the potential benefits of any nanojunction hot spots.

Finally, in Figure 3(a), we also include the $E_{\text {surf }} / E_{\text {surf }}^{0}$ ratio for a bowtie on an FP structure with realistic lossy materials. In particular, we use $\mathrm{SiO}_{2}$ as substrate $\left(n_{\mathrm{SiO}_{2}}=1.45\right)$ and $\mathrm{Si}$ as superstrate, whose refractive index at $633 \mathrm{~nm}$ is $n_{\mathrm{Si}}=3.87+$ $j 0.03$ [17]. The double-layered cavity is modified in order to fulfill the Fabry-Perot condition: $h_{1}=\lambda_{1} / 2=218.4 \mathrm{~nm}$ and $h_{2}=\lambda_{2} / 4=40.8 \mathrm{~nm}$, where $\lambda_{1}=\lambda / \sqrt{\varepsilon_{r 1}}$ and $\lambda_{2}=$ $\lambda / \sqrt{\varepsilon_{r 2}}$ are the wavelengths in the dielectrics with permittivity $\varepsilon_{r 1}=n_{\mathrm{SiO}_{2}}^{2}$ and $\varepsilon_{r 2}=\operatorname{Re}\left(n_{\mathrm{Si}}\right)^{2}$, respectively. Since the relation $\varepsilon_{r 2} \gg \varepsilon_{r 1}$ still holds (in particular $\varepsilon_{r 2} \sim 7 \varepsilon_{r 1}$ ), we expect that the resonant effect is maintained. Although the presence of losses in the silicon affects the amount of field that reaches the particles and then reduces the $E_{\text {surf }} / E_{\text {surf }}^{0}$ ratio, the enhancement is still almost constant over the range of gap values. It is clear that the general trends are not affected by dielectric losses. Therefore, in the following, we will only focus on the ideal case. This will allow us to demonstrate the general principle of operation of the structure with fewer circuitous effects.

2.2. Multiple Dimer Configuration. One of the advantages of the FP multilayered structure is that the aperture field on top of the superstrate, provided by the dipolar source, is circularly wide and linearly polarized along the source. We have shown in [11] that this aperture field remains almost unchanged when the dimer is placed in the middle of the field (except for the immediate region where the dimer is placed). This feature can be used for arranging multiple nanoparticle dimers in an array configuration. A possible geometry is shown in Figure 4 for 3 nanodisc dimers. Of course, the disc dimers can be equivalently replaced by the bowtie. The $E_{\text {surf }} / E_{\text {surf }}^{0}$ ratio is shown in Figure 5 for disc dimer (blue line) and bowtie (red line) as a function of the interelement distance $d$, for a radius $r=40 \mathrm{~nm}$ and $g a p=5 \mathrm{~nm}$. In particular, we compare the case of 3- and 5-dimer elements with the single-element configuration, which is a constant value. As expected, the enhancement of the surface field is increased significantly even though the arrays are all fed by a single dipole source. This means that wide aperture field is still strong enough over a large spatial region to excite multiple nanoparticles. This effect is particularly visible for the 3-element case, where their range of variation of the ratio $E_{\text {surf }} / E_{\text {surf }}^{0}$ in terms of $d$ is quite moderate. In the 5element case, the exterior dimers begin to fall out of the aperture field more rapidly as $d$ increases causing the field enhancement to decrease, especially for the discs when $d>$ $100 \mathrm{~nm}$. From Figure 5, it is clear that the bowtie array has an overall better field enhancement, which was also observed for the single-element case, but is amplified here for the array configuration. The larger enhancement results from the geometry of the bowtie, which supports a stronger dipolar field coupling between the bases. This creates a wider area on the sides of the bowtie surface where the field is stronger compared to the discs, which is visible in Figure 6. Here, we show the absolute value of the field distribution for arrays with 1,3 , and 5 elements, for both disc dimer and bowtie 


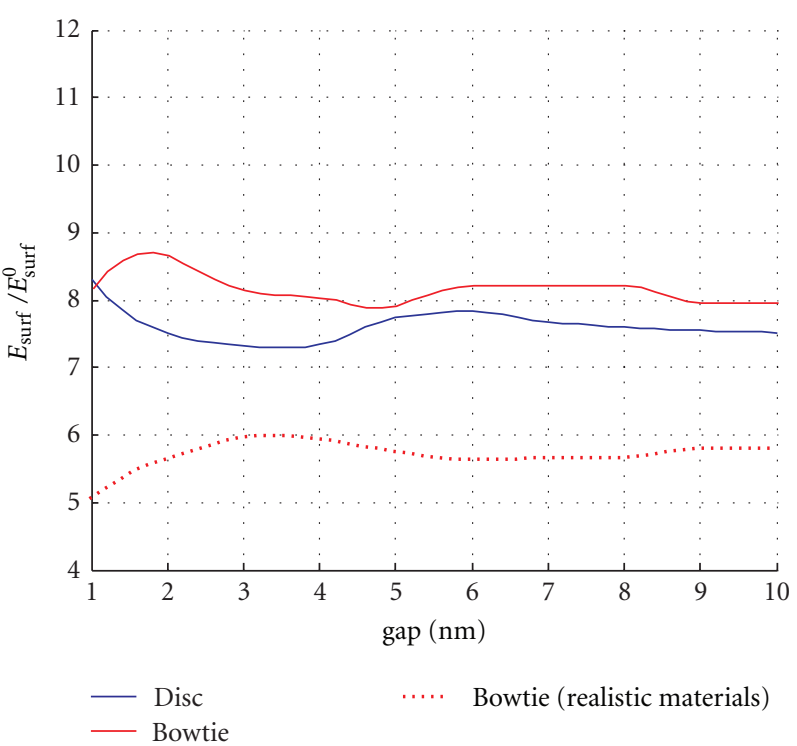

(a)

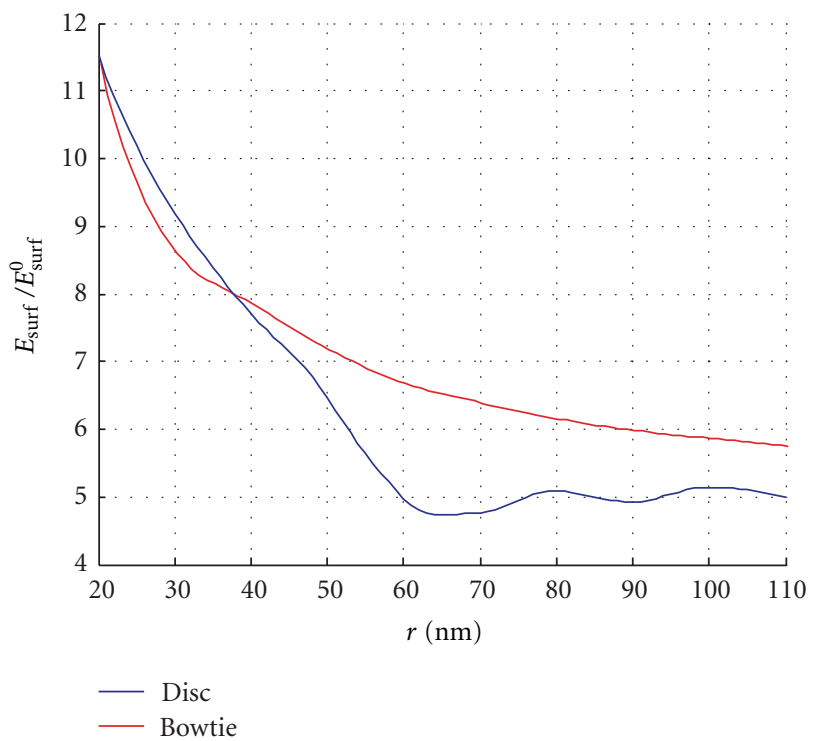

(b)

Figure 3: (a) Field enhancement for the disc dimer (blue line) and the bowtie (red line) on top of the Fabry-Perot structure as a function of the gap distance for a radius $r=40 \mathrm{~nm}$. The effect of realistic lossy materials is also shown (red dotted line) for the bowtie configuration. (b) Field enhancement as a function of the radius $r$ when $g a p=5 \mathrm{~nm}$. For both panels, the dimer and the bowtie are both oriented along the dipole source (see Figure 1).

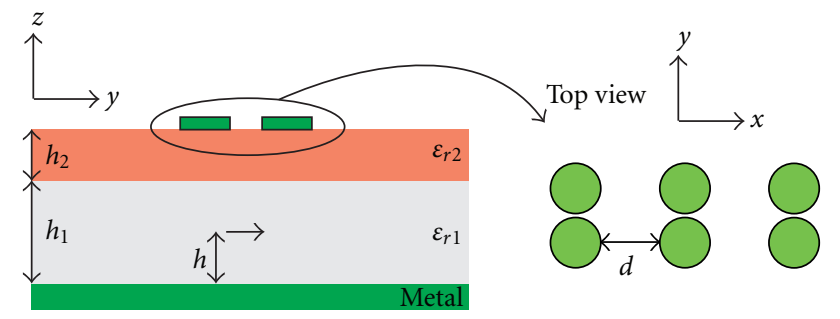

Figure 4: Multiple dimer configuration. The nanodisc dimer array can equivalently be replaced by an array of bowtie.

$(r=40 \mathrm{~nm}, g a p=5 \mathrm{~nm})$. It is clear that the disc dimers contribute to the sensing field predominantly along the side surface and the rim, while the bowtie shows a strong field distribution also on the top surface, which is more accessible to molecules.

2.3. Effect of the Source Polarization. In this section, we explore how the orientation of the dipolar source influences the enhancement of the electric field on the surface of the nanoparticles. We show here, as an example, the results for the 3-element arrays of disc dimers and bowties when the dipole is oriented along the alignment axis of the nanoparticle dimers (along $y$ ), and when the dipole is oriented perpendicularly to the dimer axis (along $x$ ). See Figure 4 for the reference system. The enhancement factor is shown in Figure 7 as a function of the interelement distance $d$. The xpol label refers to the case when the dipole is oriented perpendicularly to the main axis of the nanoparticle dimer. This result is very interesting. First,

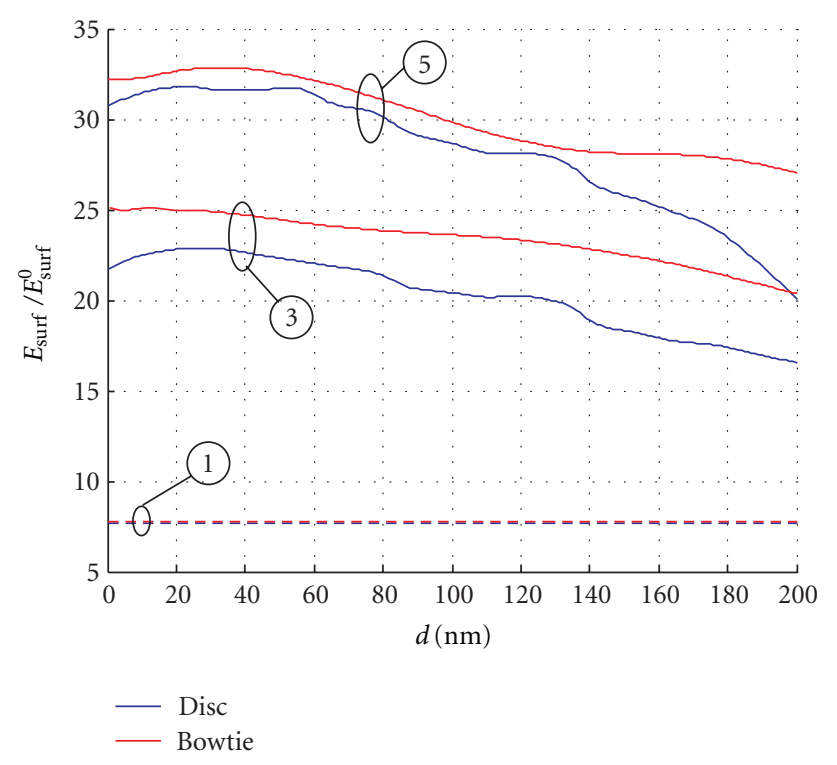

FIgURE 5: Enhancement ratio for the multiple (3 and 5) dimer configurations compared with a single dimer. In particular, the disc dimer case (blue line) and the bowtie case (red line) are plotted as a function of the interelement distance $d$, for a radius $r=40 \mathrm{~nm}$ and $g a p=5 \mathrm{~nm}$. The disc dimer and the bowtie are both oriented along the dipole source (see Figure 4).

we notice that xpol (dashed line) yields better behavior in terms of enhancement, although, for the disc dimers, the improvement is not as substantial. Nevertheless, it shows a clear trend. In this particular configuration, the xpol 


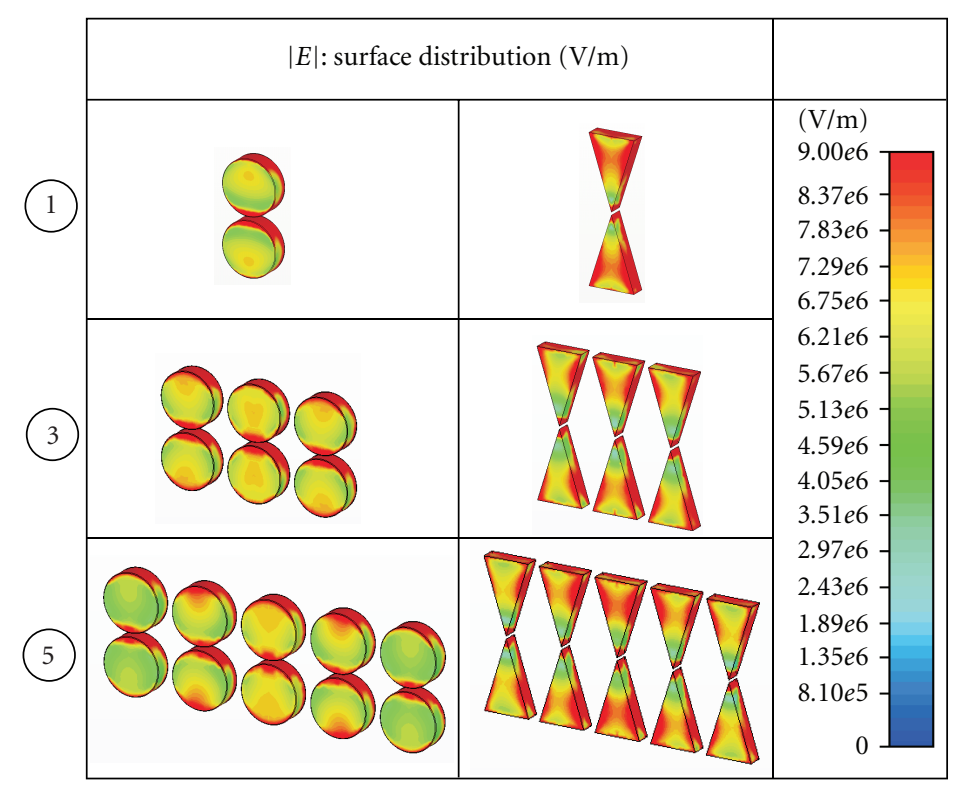

FIGURE 6: Absolute value of the field distribution for 1-, 3-, and 5-element arrays, for both the disc dimer and bowtie $(r=40 \mathrm{~nm}$, $g a p=$ $5 \mathrm{~nm}$ ). The field is generated by a dipole with length $5 \mathrm{~nm}$ and current $1 \mathrm{~A}$.

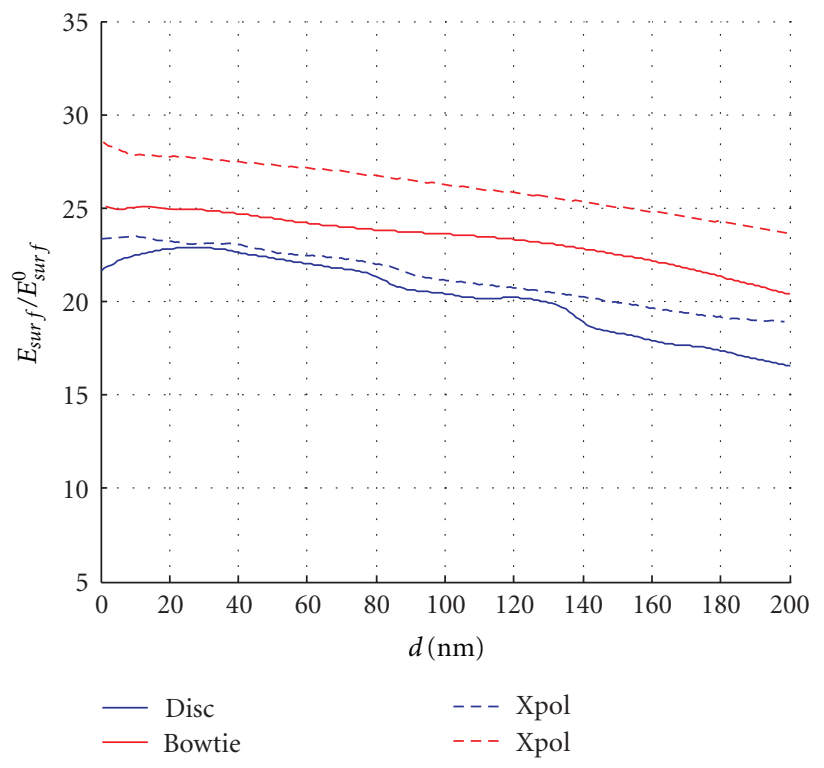

FIGURE 7: Enhancement ratio for 3-element arrays when the dipolar source is oriented along the alignment axis of the dimers (solid line) and perpendicularly to the alignment axis (dashed line), which is labelled as xpol. The disc dimer case (blue line) and the bowtie case (red line) are plotted as a function of the interelement distance $d$, for a radius $r=40 \mathrm{~nm}$ and $g a p=5 \mathrm{~nm}$.

polarization excites a greater number of equivalent dipolar field distributions over all of the array configuration. This is not particularly evident for the disc dimer but is more conspicuous for the bowtie elements. In order to accentuate this mechanism, Figure 8 shows the absolute value of the field distribution for both disc dimer and bowtie $(r=40 \mathrm{~nm}$, $g a p=5 \mathrm{~nm})$, when the dipole is oriented along the main axis or when it oriented perpendicularly. It is clear that when the dipole is perpendicular to the alignment axis, the bowtie configuration matches the aperture field distribution well, creating a stronger field on the particle surface. This particular excitation facilitates interelement coupling between tips of adjacent dimers yielding a more robust field. This result suggests that the FP structure is quite flexible to different nanoparticle geometries and configurations, since the polarization change can be used as an advantage in the SERS gain optimization process. This will be an area of future study.

\section{Conclusions}

We have investigated the sensing properties of a FabryPerot structure coupled to plasmonic nanoparticles. The Fabry-Perot structure is composed of a double layer of dielectric materials - a superstrate with a high permittivity placed on a substrate with very low permittivity. This substrate/superstrate structure has been demonstrated to establish a resonance condition, which is here used to maximize the aperture field on top of the superstrate, where the nanoparticles are located for sensing. The field is generated by a quantum emitter, treated here as a dipole, residing in the substrate. We have examined two different nanoparticle shapes, specifically disc dimers and bowtie nanostructures. A figure of merit has been defined, which allows the calculation of SERS gain based on the average surface field of the nanoparticles. We have shown that an array configuration of disc dimers and bowties can effectively increase the SERS gain, still within the excitation of a single emitter. We have also investigated how this structure can be used under different polarization conditions and find that good field enhancements can be obtained for different 


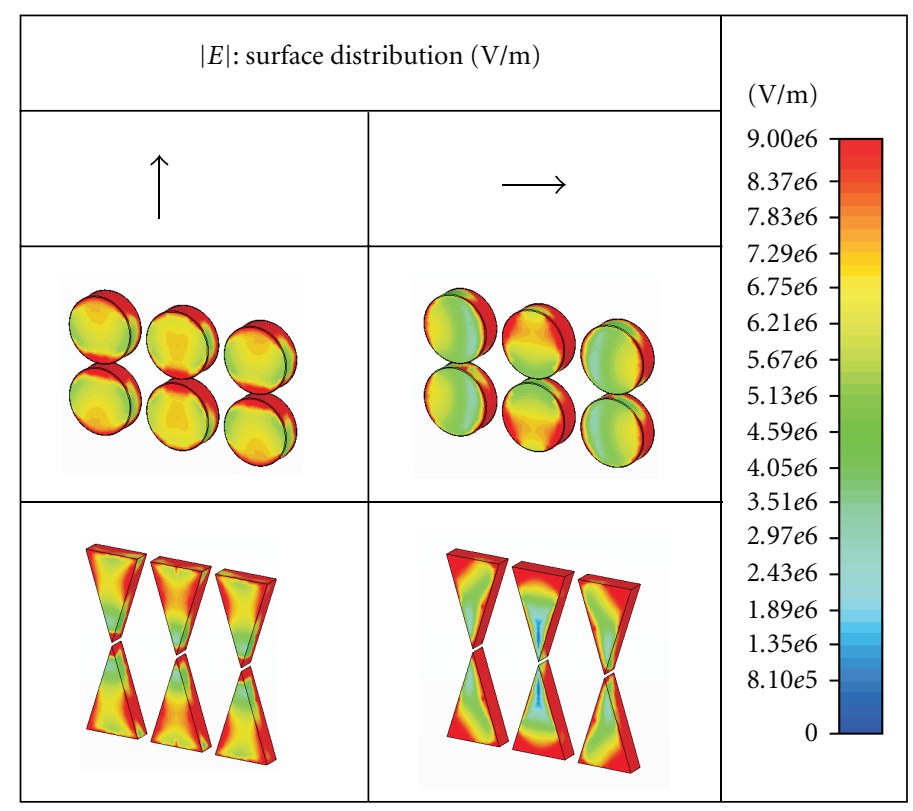

Figure 8: Absolute value of the field distribution for 3-element arrays of both disc dimers and bowties $(r=40 \mathrm{~nm}, g a p=5 \mathrm{~nm})$ when the dipolar field is oriented along or perpendicular to the alignment axis of the dimers. The field is generated by a dipole with length $5 \mathrm{~nm}$ and current $1 \mathrm{~A}$.

orientations of the dipolar source. This is a promising result, suggesting that other nanoparticle geometries and new array arrangements could be incorporated without detrimental effects to the sensing process.

\section{Acknowledgment}

This material is based upon work supported by the Department of Energy under Award Number DE-SC0006922.

\section{References}

[1] S. Nie and S. R. Emory, "Probing single molecules and single nanoparticles by surface-enhanced Raman scattering," Science, vol. 275, no. 5303, pp. 1102-1106, 1997.

[2] K. Kneipp, Y. Wang, H. Kneipp et al., "Single molecule detection using surface-enhanced Raman scattering (SERS)," Physical Review Letters, vol. 78, no. 9, pp. 1667-1670, 1997.

[3] R. Aroca, Surface-Enhanced Vibrational Spectroscopy, John Wiley and Son, Chichester, UK, 2006.

[4] H. C. Kim and X. Cheng, "SERS-active substrate based on gap surface plasmon polaritons," Optics Express, vol. 17, no. 20, pp. 17234-17241, 2009.

[5] L. C. T. Shoute, A. J. Bergren, A. M. Mahmoud, K. D. Harris, and R. L. McCreery, "Optical interference effects in the design of substrates for surface-enhanced raman spectroscopy," Applied Spectroscopy, vol. 63, no. 2, pp. 133140, 2009.

[6] M. J. Banholzer, J. E. Millstone, L. Qin, and C. A. Mirkin, "Rationally designed nanostructures for surface-enhanced Raman spectroscopy," Chemical Society Reviews, vol. 37, no. 5, pp. 885-897, 2008.

[7] A. Campion, J. E. Ivanecky III, C. M. Child, and M. Foster, "Chemical enhancement in surface-enhanced raman scattering," Journal of the American Chemical Society, vol. 117, no. 47, pp. 11807-11808, 1995.

[8] P. Kambhampati, C. M. Child, M. C. Foster, and A. Campion, "On the chemical mechanism of surface enhanced Raman scattering: experiment and theory," Journal of Chemical Physics, vol. 108, no. 12, pp. 5013-5026, 1998.

[9] K. L. Kelly, E. Coronado, L. L. Zhao, and G. C. Schatz, “The optical properties of metal nanoparticles: the influence of size, shape, and dielectric environment," Journal of Physical Chemistry B, vol. 107, no. 3, pp. 668-677, 2003.

[10] R. Boyack and E. C. Le Ru, "Investigation of particle shape and size effects in SERS using T-matrix calculations," Physical Chemistry Chemical Physics, vol. 11, no. 34, pp. 7398-7405, 2009.

[11] A. Polemi and K. L. Shuford, "Fabry-Perot effect on dimer nanoantennas," Photonics and Nanostructures, vol. 10, no. 1, pp. 36-45, 2012.

[12] N. G. Alexopoulos and D. R. Jackson, "Fundamental superstrate (cover) effects on printed circuit antennas," IEEE Transactions on Antennas and Propagation, vol. 32, no. 8, pp. 807-816, 1984.

[13] D. R. Jackson and N. G. Alexopoulos, "Gain enhancement methods for printed circuit antennas," IEEE Transactions on Antennas and Propagation, vol. AP-33, no. 9, pp. 976-987, 1985.

[14] D. R. Jackson and A. A. Oliner, "Leaky-wave analysis of the high-gain printed antenna configuration," IEEE Transactions on Antennas and Propagation, vol. 36, no. 7, pp. 905-910, 1988.

[15] L. Allen and J. H. Eberly, Optical Resonance and Two-Level Atoms, Dover Publications, Dover, Del, USA, 1987.

[16] A. Polemi and S. Maci, "On the polarization properties of metamaterial lenses," IEEE Antennas and Wireless Propagation Letters, vol. 5, no. 1, pp. 306-310, 2006.

[17] E. D. Palik, Handbook of Optical Constants of Solids, Academic Press, Orlando, Fla, USA, 1985. 
[18] A. Polemi, S. M. Wells, N. V. Lavrik, M. J. Sepaniak, and K. L. Shuford, "Local field enhancement of pillar nanosurfaces for SERS," Journal of Physical Chemistry C, vol. 114, no. 42, pp. 18096-18102, 2010.

[19] A. Polemi, S. M. Wells, N. V. Lavrik, M. J. Sepaniak, and K. L. Shuford, "Dispersion characteristics in Diskon-Pillar array nanostructures for surface enhanced Raman spectroscopy," The Journal of Physical Chemistry C, vol. 115, no. 28, pp. 13624-13629, 2011.

[20] "CST Computer Simulation Technology AG," Darmstadt, Germany, 2011, http://www.cst.com.

[21] T. Weiland, "Discretization Method for the Solution of Maxwell's Equations for Six-Component Fields," AEU-Archiv fur Elektronik und Ubertragungstechnik, vol. 31, no. 3, pp. 116120, 1977. 

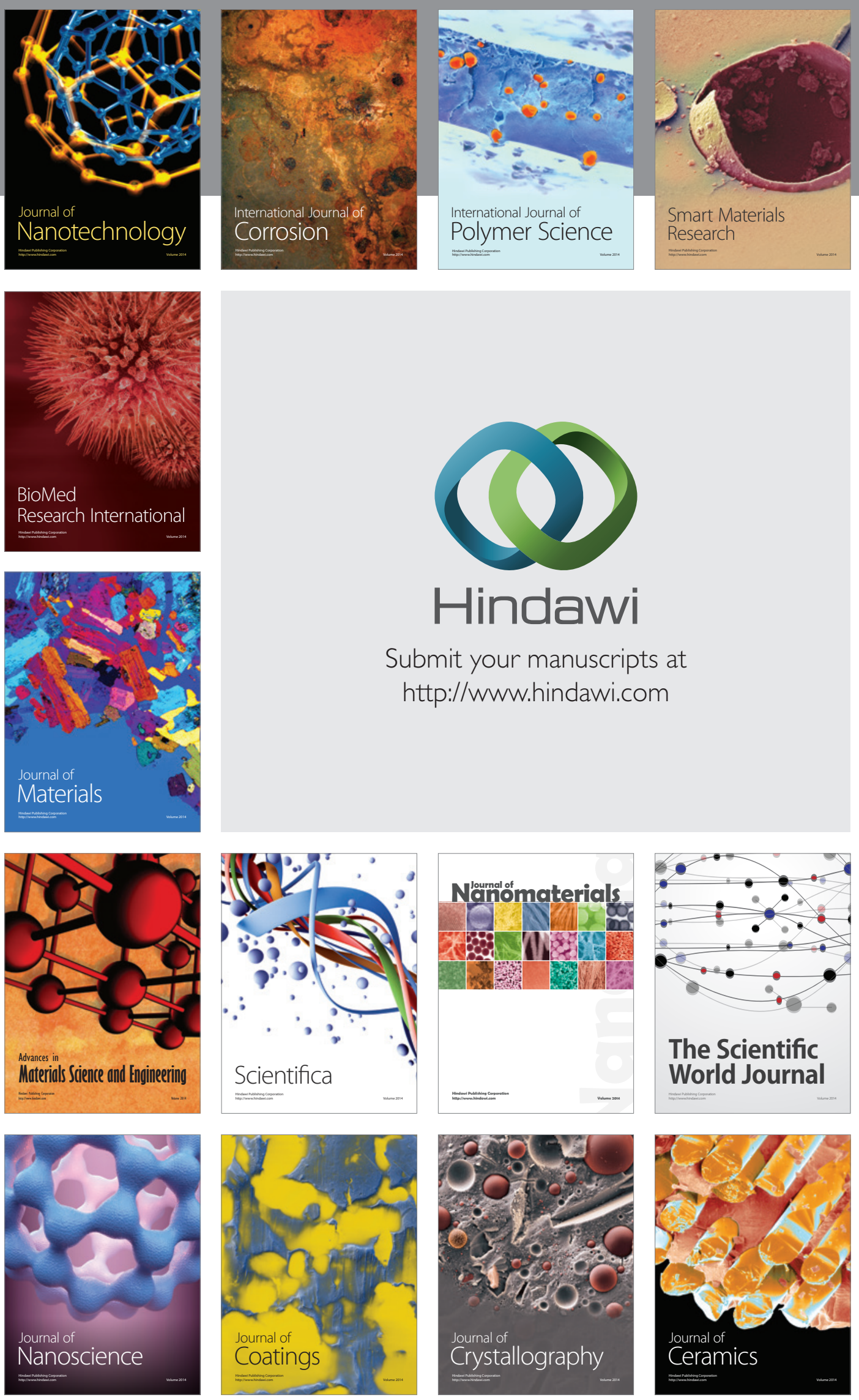

The Scientific World Journal

Submit your manuscripts at

http://www.hindawi.com

\section{World Journal}

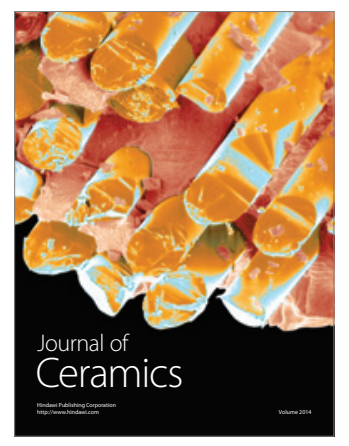

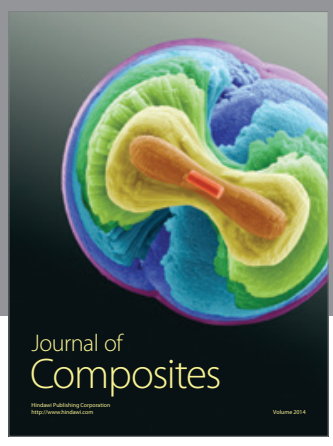
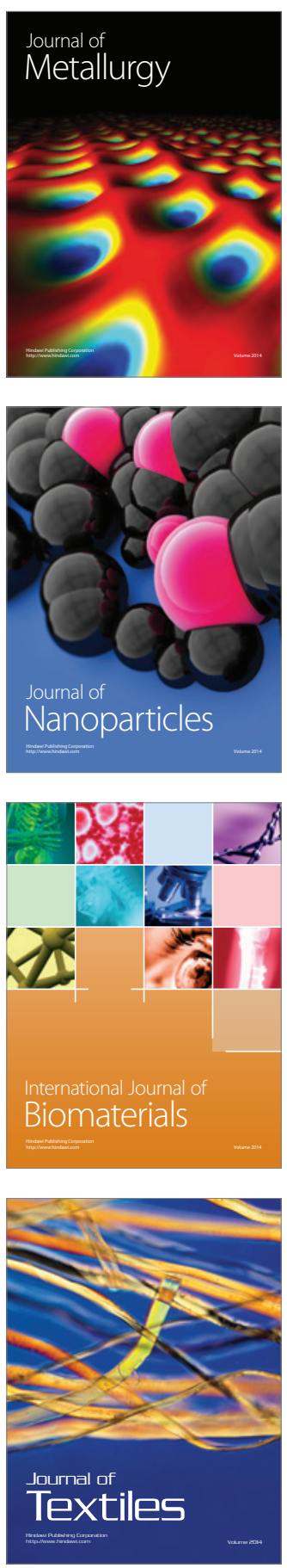